\title{
A Unified Statistical Approach to Deformation-Based Morphometry
}

\author{
M. K. Chung,* K. J. Worsley,*`† T. Paus,† C. Cherif,‡ D. L. Collins, † J . N. Giedd,§ \\ J. L. Rapoport,§ and A. C. Evanst \\ *Department of Mathematics and Statistics and †Montreal Neurological Institute, McGill University, Montréal, Québec, Canada; \\ ‡Département de Mathématiques, É cole Polytechnique Fédérale de Lausanne, Switzerland; and §Child Psychiatry Branch, \\ National Institute of Mental Health, NIH, Bethesda, Maryland 20892
}

Received August 14, 2000

\begin{abstract}
We present a unified statistical framework for analyzing temporally varying brain morphology using the 3D displacement vector field from a nonlinear deformation required to register a subject's brain to an atlas brain. The unification comes from a single model for structural change, rather than two separate models, one for displacement and one for volume changes. The displacement velocity field rather than the displacement itself is used to set up a linear model to account for temporal variations. By introducing the rate of the J acobian change of the deformation, the local volume change at each voxel can be computed and used to measure possible brain tissue growth or loss. We have applied this method to detecting regions of a morphological change in a group of children and adolescents. Using structural magnetic resonance images for $\mathbf{2 8}$ children and adolescents taken at different time intervals, we demonstrate how this method works. 2001 Academic Press
\end{abstract}

Key Words: volume change; volumetry; brain growth; morphometry; atrophy; deformation; brain development.

\section{INTRODUCTION}

Temporally varying morphological differences in the brain have been examined primarily by MRI-based volumetry. Classical MRI-based volumetry requires segmentation of the identical region of interest, either manually or by spatial normalization, in two MR images taken at different times $t_{1}$ and $t_{2}$. Then the total volumes $V_{1}$ and $V_{2}$ of the homologous regions are calculated by counting the total number of voxels. Afterward, the vol ume variation $\Delta \mathrm{V}=\mathrm{V}_{2}-\mathrm{V}_{1}$ is used as an index of morphological changes (Giedd et al., 1996a; Rajapakse et al., 1996; Reiss et al., 1996; Thirion and Calmon, 1999).

As a part of deformation-based morphometry, a new technique called deformation-based volumetry is emerging; this method does not require segmentation of a priori regions of interest (Davatzikos, 1999; Ash- burner and Friston, 2000). In deformation-based volumetry, the J acobian of the deformation field that is required to register one brain to another is used to detect volumetric changes. By definition, the J acobian of the deformation is the volume of the unit-cube after the deformation. Assuming that one can find the deformation field at any voxel, volume change can be detected at a voxel level. So the advantage of this technique over the classical MRI-based volumetry is that it does not require a priori knowledge of the region of interest to perform the morphological analysis. Moreover, the deformation-based volumetry improves the power of detecting the regions of volume change within the limits of the accuracy of the registration algorithm. These two advantages of the deformation-based volumetry over the standard MRI-based volumetry have also been noted by Davatzikos (1999) and Ashburner and Friston (2000).

Because the deformation-based morphometry (DBM) is a relatively new method, very few morphological studies have used the J acobian for local volume change. Davatzikos et al. (1996) used the J acobian of the $2 \mathrm{D}$ deformation field as a measure of local area change in 2D cross-sections of the corpus callosum to test gender-specific shape differences. Thompson and Toga (1999) applied the J acobian of 3D deformations as a measure of the regional growth of the corpus callosum. Also volume dilatation, which is the first-order approximation of the J acobian, has been used instead of the J acobian itself to measure local volume change. Thirion and Calmon (1999) used the divergence of the displacement vector field, which is equivalent to the dilatation, for detecting growth of brain tumors. Thompson et al. (2000) used local rates of dilatation, contraction, and shearing from the deformation field to detect morphological changes in brain development.

There has also been a parallel development in detecting morphological changes without volumetry using Hotelling's $\mathrm{T}^{2}$ statistic for the displacement field (Thompson et al., 1997; J oshi, 1998; Collins et al., 1998; Gaser et al., 1999; Cao and Worsley, 1999). Although it 
$\mathbf{a}$

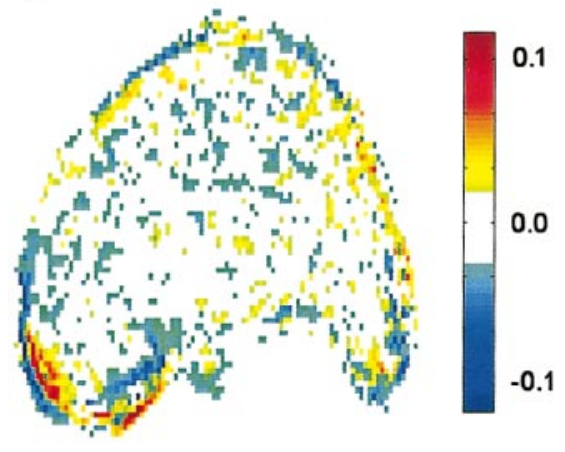

b

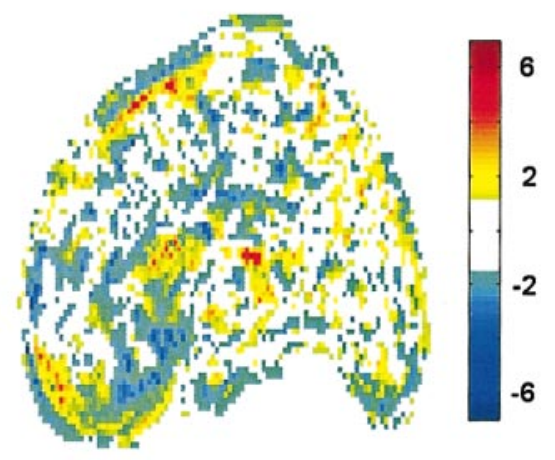

d

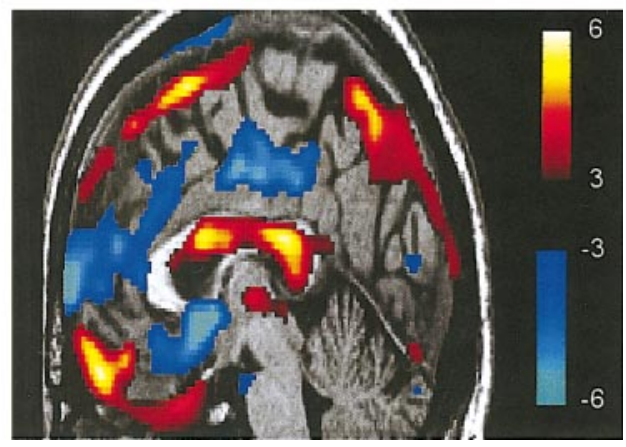

FIG. 1. The statistical analysis of local volume change data on the midsagittal section. (a) The sample mean dilatation rate $\mathrm{M}_{\text {volume. }}$ It gives an incorrect impression that the local volume change only occurs near the outer cortical boundaries due, perhaps, to registration error. (b) t map of local volume change. Local maxima appear around the corpus callosum. A lot of noise on the cortical boundaries disappears. (c) t map of local volume change after $10-\mathrm{mm}$ Gaussian kernel smoothing. The smoothing is applied directly to the displacement fiel ds and the signal-to-noise ratio improves. (d) Thresholded t map superimposed on the midsagittal section of the atlas brain. The corpus callosum shows volume increase. When the corrected threshold of $\mathrm{t}>6.5$ is applied, most of the red regions disappears except for the local maximum in the isthmus and splenium of the corpus callosum.

seems that there are many different ways of detecting morphological changes in deformation-based morphometry, a translation, a rotation, and a strain are sufficient for detecting a relatively small displacement and, in turn, for characterization of morphological changes over time.

In this paper, we present a unified statistical framework for detecting brain tissue growth and loss is temporally varying brain morphology. As an illustration, we will demonstrate how the method can be applied to detecting regions of tissue growth and loss in brain images longitudinally collected in a group of children and adolescents.

\section{METHODS}

\section{Statistical Model}

Unlike other brain morphological studies that try to characterize the structural variabilities among different individuals of similar age groups, morphological studies of temporally varying brain structure have an extra temporal dimension. Therefore, a different ap- proach to morphometry is required to fully understand the spatiotemporal complexity of brain development.

Let $U(x, t)=\left(U_{1}, U_{2}, U_{3}\right)$ be the 3D displacement vector field required to move the structure at position $x=\left(x_{1}, x_{2}, x_{3}\right) \in \mathbb{R}^{3}$ and at the reference time 0 of a subject brain to the corresponding position after timet. Thus the structure at $x$ deforms to $x+U(x, t)$ with respect to a fixed reference coordinate. The displacement field $U(x, t)$ at fixed time $t$ is usually estimated via volume-based nonlinear registration techniques on two images taken at time 0 and at time t. Then we propose to test the following stochastic model of brain development,

$$
\frac{\partial \mathrm{U}}{\partial \mathrm{t}}(\mathrm{x}, \mathrm{t})=\mathrm{L}(\mathrm{U})+\Sigma^{1 / 2}(\mathrm{x}) \epsilon(\mathrm{x}),
$$

where $L$ is a partial differential operator involving spatial components and $\Sigma(x)$ is the $3 \times 3$ symmetric positive-definite covariance matrix, which allows correlations between components of the deformations and depends on the spatial coordinates $x$ only. Since $\Sigma$ is 

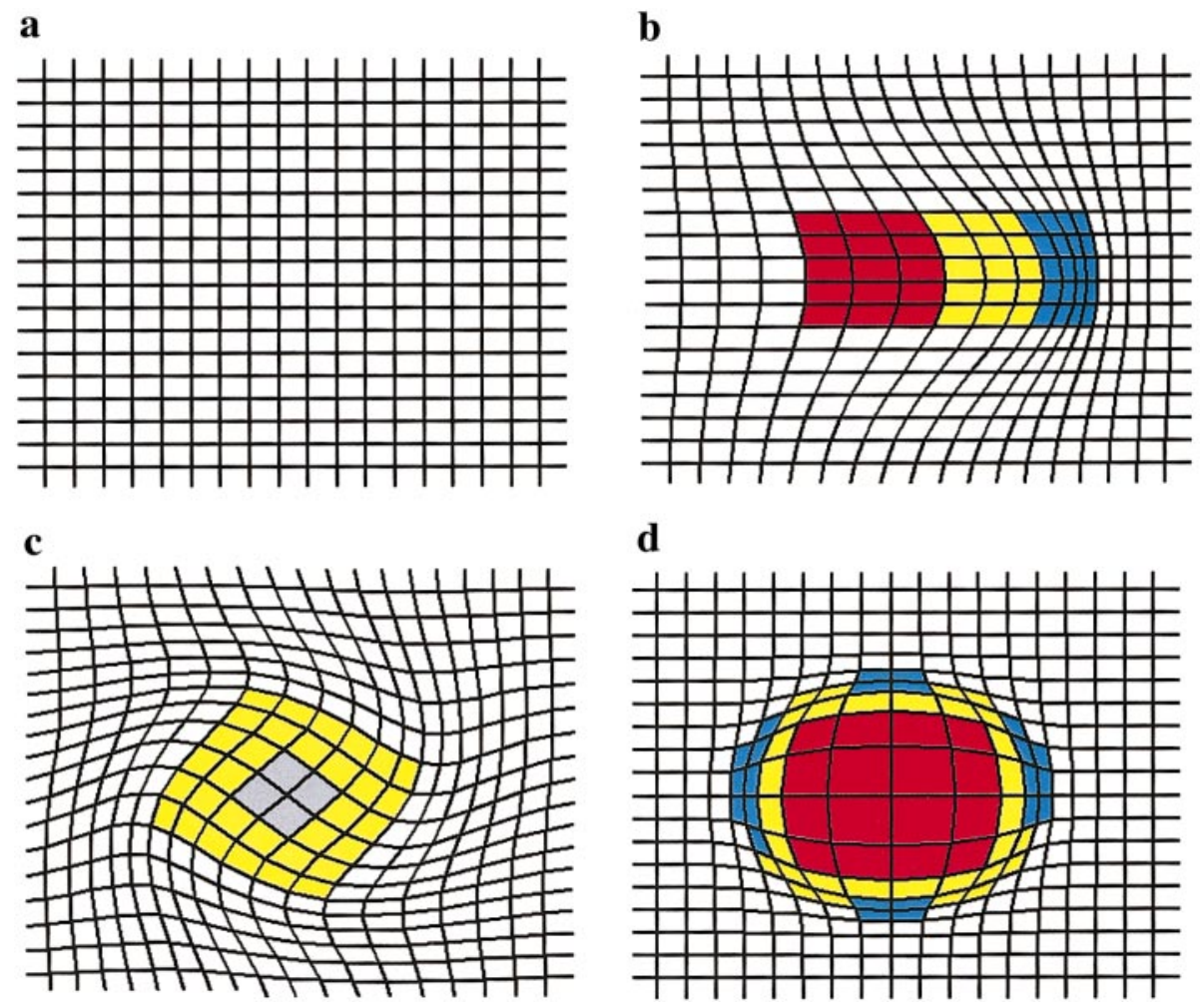

FIG. 2. Square grid under translation, rotation, and volume change. Red, volume increase; blue, volume decrease; gray, rotation; yellow, translation. (a) Square grid under no deformation. (b) Horizontal translation caused by local volume increase on the left side. (c) $45^{\circ} \mathrm{cl}$ ockwise rotation. The rotation induces the outer region of the center of the rotation to translate. (d) Volume expansion in the middle causes the grid to radially translate outward.

symmetric positive-definite, the square root of $\Sigma$ always exists. The components of the error vector $\epsilon$ are independent and identically distributed as smooth stationary Gaussian random fields with zero mean and unit standard deviation. The error structure $\Sigma^{1 / 2} \epsilon$ was first introduced in Worsley (1996) and Cao and Worsley (1999). An equation of type (1) is called a stochastic evolution equation and it models how the structure evolves over time. Any smooth morphological change can be completely described by (1) within the bound set by the error structure $\Sigma^{1 / 2} \epsilon$. Modeling the rate of change as a differential equation originates from $\mathrm{New}$ ton. If the deformation is assumed to follow a diffusing behavior, then $\mathrm{L}$ can be chosen as the Laplacian operator

$$
L=\sigma^{2}\left(\frac{\partial^{2}}{\partial x_{1}^{2}}+\frac{\partial^{2}}{\partial x_{2}^{2}}+\frac{\partial^{2}}{\partial x_{3}^{2}}\right)
$$

If the morphological changes are assumed to follow a fluid dynamics model, $L$ becomes a Navier-Stokes operator given in Landau and Lifshitz (1989).

Longitudinal analysis based on (1) is essentially the inverse problem of brain registration. This analysis tries to determine the partial differential operator $\mathrm{L}$ with given displacement fields. On the other hand, in brain registration, the objective is to find the displacement field $U$ that matches homologous points between two images based on minimizing a cost function or actually solving partial differential equations. The most widely used physical models that have been used in brain registration are elastic deformations and fluid dynamics models (Christensen et al., 1993; Thompson et al., 1999; Davatzikos, 1999; Gee and Bajcsy, 1999). Suppose that the displacement field $U$ is obtained as a solution of the elastic deformation equation given by

$$
\frac{\partial \mathrm{U}}{\partial \mathrm{t}}=\mathrm{L}_{\text {elastic }}(\mathrm{U})+\Sigma^{1 / 2} \epsilon
$$

where the elastic operator

$$
\mathrm{L}_{\text {elastic }}(\mathrm{U})=\lambda_{1} \nabla^{2} \mathrm{U}+\lambda_{2} \nabla(\nabla \cdot \mathrm{U})+\mathrm{F}
$$

is defined in Warfield et al. (1999). Then using this displacement field $U$ as given data, we try to estimate (1) which minimizes a certain error criterion based on $\Sigma^{1 / 2} \epsilon$. Then the best estimator of $L$ is heavily biased toward the prior operator $L_{\text {elastic. }}$ It indicates that the estimation of (1) should be based on an image registra- 
tion method that does not assume an a priori physical model or on an empirical Bayesian framework. We will use intensity-based registration algorithms that do not have explicit physical model assumptions to warp one brain to another (Collins et al., 1995; Ashburner et al., 1997), but there should be further comparative studies of the different image registration methods to draw any general conclusions.

It can be assumed that, in the case of morphological changes occurring in a healthy brain over a relatively short period of time, deformation occurs continuously and smoothly, so the higher order temporal derivatives of the displacement $U$ are relatively small compared to the displacement itself. In such a case, the first-order approximation to $L(U)$ is sufficient to capture most of the morphological variabilities over time. Therefore, we approximate $\partial \mathrm{U} / \partial \mathrm{t}$ with only a first-order term $\mu_{0}(\mathrm{x})$ which is constant over time; i.e.,

$$
\frac{\partial \mathrm{U}}{\partial \mathrm{t}}(\mathrm{x}, \mathrm{t})=\mu_{0}(\mathrm{x})+\Sigma^{1 / 2}(\mathrm{x}) \epsilon(\mathrm{x})
$$

By taking the expectation $\mathbb{E}$ on the both sides of (2), we see that $\mu_{0}=\mathbb{E} \partial \mathrm{U} / \partial \mathrm{t}$, the mean displacement rate. Under the linear model (2), the problem of detecting local displacement can be solved with a simple hypothesis test:

$$
\mathrm{H}_{0}: \mu_{0}(\mathrm{x})=0 \text { vs } \mathrm{H}_{1}: \mu_{0}(\mathrm{x}) \neq 0 \text {. }
$$

If one wishes to see the convexity of the growth curve, an additional second-order term is needed in (2). Unlike estimating the first-order linear term $\mu_{0}$, the problem of estimating the second-order nonlinear term requires a large amount of data to have a statistically stable result due to intrasubject variabilities across spatial and temporal dimensions. In this paper, we have limited our discussion to the detection of the first-order morphological changes and we will not attempt to analyze the full model (1).

\section{Detecting Local Displacement}

We are interested in detecting regions with statistically significant changes in displacement using the linear model (2) under a Gaussian error structure. This is a standard multivariate statistical inference problem and solved using Hotel ling's $\mathrm{T}^{2}$ statistic (Thompson et al., 1997; J oshi, 1998; Gaser et al., 1999; Cao and Worsley, 1999).

Let $U^{j}\left(x, t_{j}\right)$ be the 3D displacement vector field required to deform the structure at the reference time 0 of the brain of subject $j$ to the corresponding homologous position after time $t_{j}$. Let

$$
V^{j}(x)=\frac{U^{j}\left(x, t_{j}\right)}{t_{j}}
$$

be the displacement velocity of subject j. Then the sample mean displacement velocity $\bar{V}$ is given by

$$
\bar{V}(x)=\frac{1}{n} \sum_{j=1}^{n} V^{j}(x),
$$

while the sample covariance matrix $C$ of the displacement velocity is given by

$$
C(x)=\frac{1}{n-1} \sum_{j=1}^{n}\left(V^{j}(x)-\bar{V}(x)\right)\left(V^{j}(x)-\bar{V}(x)\right)^{t}
$$

where the superscript $t$ denotes the matrix transpose. Then Hotelling's $\mathrm{T}^{2}$ field $\mathrm{H}(\mathrm{x})$ is defined as

$$
H(x)=n \bar{V}^{t}(x) C^{-1}(x) \bar{V}(x)
$$

At each voxel $x$, under the hypothesis of no mean displacement velocity, i.e., $\mu_{0}(x)=0, H(x)$ is distributed as a multiple of an $\mathrm{F}$ distribution with $(3, \mathrm{n}-3)$ degrees of freedom; i.e.,

$$
H(x) \sim 3 \frac{n-1}{n-3} F_{3, n-3}
$$

Then the $\mathrm{P}$ value of the maxima of $\mathrm{H}(\mathrm{x})$, which corrects for searching across a whole brain volume, is used to localize the region of statistically significant structural displacement (Cao and Worsley, 1999). As pointed out in Ashburner and Friston (2000), Hotelling's $T^{2}$ statistic based on the displacement field does not directly localize regions within different structures, but rather identifies brain structures that have translated to different positions. It measures relative position of two particular voxels before and after the deformation. Therefore, in the context of temporally varying brain morphology where the brain volume change is an important concern, the statistic based on the displacement field should be taken as an indirect measure of brain growth. The more direct morphological criterion that corresponds to the actual brain tissue growth or loss is the J acobian of the deformation field, which we will look at the next section.

\section{Detecting Local Volume Change}

The deformation in the Lagrangian coordinate system, i.e., fixed coordinate system at time $t$, is

$$
x \rightarrow x+U(x, t)
$$

The local volume change of the deformation in the neighborhood of a point $x$ and at time $t$ is determined by the J acobian J, which is defined as $\mathrm{J}(\mathrm{x}, \mathrm{t})=\operatorname{det}(\mathrm{I}+$ 
$\partial \mathrm{U} / \partial \mathrm{x})$, where I denotes an identity matrix and $\partial \mathrm{U} / \partial \mathrm{x}$ is the $3 \times 3$ displacement gradient matrix of $U$ given by

$$
\frac{\partial U}{\partial x}(x, t)=\left(\begin{array}{lll}
\frac{\partial U_{1}}{\partial x_{1}} & \frac{\partial U_{1}}{\partial x_{2}} & \frac{\partial U_{1}}{\partial x_{3}} \\
\frac{\partial U_{2}}{\partial x_{1}} & \frac{\partial U_{2}}{\partial x_{2}} & \frac{\partial U_{2}}{\partial x_{3}} \\
\frac{\partial U_{3}}{\partial x_{1}} & \frac{\partial U_{3}}{\partial x_{2}} & \frac{\partial U_{3}}{\partial x_{3}}
\end{array}\right) .
$$

The component $\partial \mathrm{U}_{\mathrm{j}} / \partial \mathrm{x}_{\mathrm{i}}$ is called the displacement tensor and, in tensor-based morphometry (Ashburner and Friston, 2000), these nine components form scalar fields used to measure the second-order morphological variabilities. Note that local translation captures the first-order morphological variability. A statistical model for the displacement gradient $\partial \mathrm{U} / \partial \mathrm{x}$ can be directly derived from (1) by taking the partial derivative with respect to the spatial coordinates $x$. Hence, by modeling the morphological changes in the random fields (Adler, 1981; Worsley et al., 1996), the situation of having two possibly incompatible statistical models on the displacement $U$ and the displacement gradient $\partial \mathrm{U} / \partial \mathrm{x}$ can be avoided. In our unified statistical modeling approach using (1), all possible statistical distributions of morphological test criteria can be directly derived and easily manipulated from (1).

Since the J acobian J measures the volume of the deformed unit-cube after timet, the rate of the change of the J acobian J , i.e., $\partial \mathrm{J} / \partial \mathrm{t}$, is the rate of the local volume change. In brain imaging, a voxel can be considered the unit-cube; therefore, $\partial \mathrm{J} / \partial \mathrm{t}(\mathrm{x})$ essentially measures the change in the volume of voxel $x$ after the deformation.

Expanding the $\mathrm{J}$ acobian $\mathrm{J}$, we get

$$
\begin{aligned}
\mathrm{J} & =\operatorname{det}(I+\nabla U) \\
& =1+\operatorname{tr}(\nabla U)+\operatorname{detr}_{2}(\nabla U)+\operatorname{det}(\nabla U),
\end{aligned}
$$

where $\operatorname{detr}_{2}(\nabla U)$ is the sum of $2 \times 2$ principal minors of $\nabla U$. For relatively small displacements, which is the case in brain development, we may neglect the higher order terms and get $\mathrm{J} \approx 1+\operatorname{tr}(\nabla U)$. Taking the partial derivative with respect to the temporal coordinatet, we get

$$
\begin{aligned}
\frac{\partial \mathrm{J}}{\partial \mathrm{t}} & \approx \frac{\partial^{2} \mathrm{U}_{1}}{\partial \mathrm{t} \partial \mathrm{X}_{1}}+\frac{\partial^{2} \mathrm{U}_{2}}{\partial \mathrm{t} \partial \mathrm{X}_{2}}+\frac{\partial^{2} \mathrm{U}_{3}}{\partial \mathrm{t} \partial \mathrm{X}_{3}} \\
& =\frac{\partial}{\partial \mathrm{t}}(\nabla \cdot \mathrm{U}) \\
& =\nabla \cdot\left(\frac{\partial \mathrm{U}}{\partial \mathrm{t}}\right)
\end{aligned}
$$

where $\nabla \cdot$ is the divergence operator. In elastic theory, the volume dilatation is defined as $\Theta_{\text {volume }}(x)=\nabla \cdot U$ (Marsden and Hughes, 1983). Therefore, the rate of the J acobian change is approximately the rate of the volume dilatation change for relatively small displacements, i.e.,

$$
\frac{\partial \mathrm{J}}{\partial \mathrm{t}} \approx \frac{\partial}{\partial \mathrm{t}} \Theta_{\text {volume }}(\mathrm{x})=\Lambda_{\text {volume }}(\mathrm{x}),
$$

where we term $\Lambda_{\text {volume }}$ to be the volume dilatation rate. Since derivatives of a Gaussian field and the sum of components of a multivariate Gaussian field are again Gaussian field, from (2), we have a linear model on the volume dilatation rate $\Lambda_{\text {volume }}$ given by

$$
\Lambda_{\text {volume }}(\mathrm{x})=\lambda_{\text {volume }}(\mathrm{x})+\epsilon_{\text {volume }}(\mathrm{x}),
$$

where $\lambda_{\text {volume }}$ is the mean volume dilatation rate and $\epsilon_{\text {volume }}$ is a Gaussian random fiel d with zero mean. When $\lambda_{\text {volume }}(x)=0$ in the neighborhood of $x$, the deformation is incompressible so there is no volume change. However, if $\lambda_{\text {volume }}(x)>0$, the volume increases while $\lambda_{\text {volume }}(\mathrm{x})<0$, the volume decreases after the deformation. In certain registration algorithms, the J acobian J is forced to be larger than a certain threshold to ensure the homologous correspondence between two brains (Christensen et al., 1997). When such a registration algorithm is used, the power of detecting the region of statistically significant volume change may be reduced. Statistical inference on the linear model (4) is easier than that of (2) since it is a univariate Gaussian. To detect statistically significant local volume change, the $T$ random field with its $P$ value of the maximum field can be used (Worsley et al., 1994).

Let $\Theta_{\text {volume }}^{j}$ denote the volume dilatation of the displacement $U^{j}=\left(U_{1}^{j}, U_{2}^{j}, U_{3}^{j}\right)$ for subject $j$ after time $t_{j}$. Then the volume dilatation rate or growth rate $\Lambda_{\text {volume }}^{\mathrm{j}}$ of subject $\mathrm{j}$ is

$$
\Lambda_{\text {volume }}^{j}=\frac{1}{t_{j}} \Theta_{\text {volume }}^{j}=\frac{1}{t_{j}}\left(\frac{\partial U_{1}^{j}}{\partial x_{1}}+\frac{\partial U_{2}^{j}}{\partial x_{2}}+\frac{\partial U_{3}^{j}}{\partial x_{3}}\right) .
$$

In the actual numerical implementation, the displacement tensor $\partial \mathrm{U}_{i}^{\mathrm{j}} / \partial \mathrm{x}_{\mathrm{i}}$ can be computed by the finite difference on rectangular grid. For example, at voxel position $x=\left(x_{1}, x_{2}, x_{3}\right)$,

$$
\frac{\partial U_{1}^{j}}{\partial x_{1}} \approx \frac{U_{1}^{j}\left(x_{1}+\delta x_{1}, x_{2}, x_{3}\right)-U_{1}^{j}\left(x_{1}, x_{2}, x_{3}\right)}{\delta x_{1}},
$$

where $\delta \mathrm{x}_{1}$ is the length of the edge of a voxel along the $\mathrm{x}_{1}$ axis. Then the $\mathrm{T}$ random field is defined as 


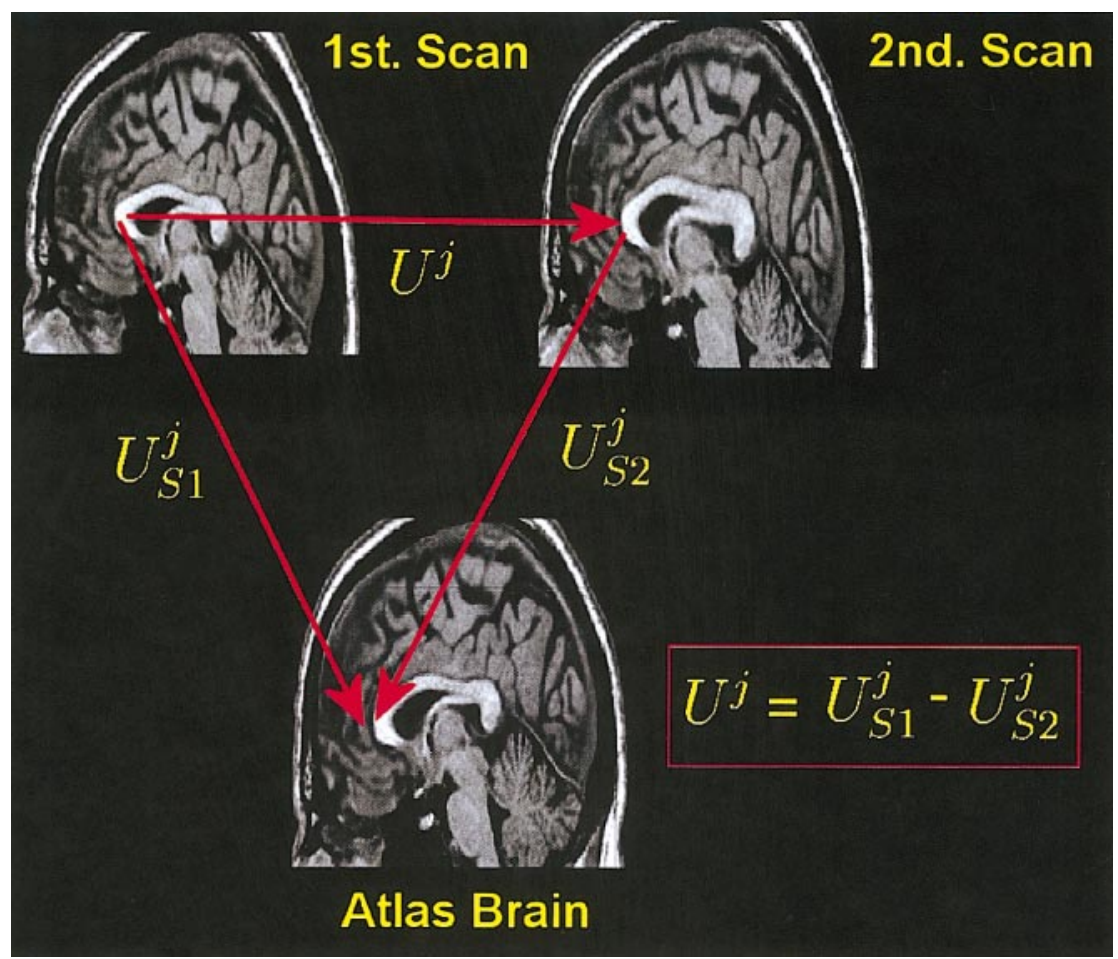

FIG. 3. The procedure for computing the displacement vector field $U^{j}$ for subject j. (1) Compute the displacement field $U_{S 1}^{j}$ from the first scan registered onto the atlas brain $\Omega_{\text {atlas. }}$ (2) Compute the displacement field $U_{\mathrm{s} 2}^{\mathrm{j}}$ from the second scan registered onto the atlas brain $\Omega_{\text {atlas }}$. (3) Compute the difference $U^{j}=U_{S 1}^{j}-U_{s 2}^{j}$, which is then automatically defined at each voxel $x \in \Omega_{\text {atlas. }}$.

$$
\mathrm{T}(\mathrm{x})=\sqrt{\mathrm{n}} \frac{\mathrm{M}_{\text {volume }}(\mathrm{x})}{\mathrm{S}_{\text {volume }}(\mathrm{x})},
$$

where $\mathbf{M}_{\text {volume }}$ and $\mathrm{S}_{\text {volume }}$ are the sample mean and standard deviation of $\Lambda_{\text {volume. }}^{\mathrm{j}}$ Under the assumption of no local volume change at $x$, i.e., $\lambda_{\text {volume }}(x)=0, T(x) \sim$ $t_{n-1}$, a Student $t$ distribution with $n-1$ degrees of freedom. As we shall see under Results, the sample mean dilatation rate $M_{\text {volume }}$ does not provide accurate information about where the brain growth is dominant but the $T$ field does (Fig. 1). Then the $P$ value of the maxima of $\mathrm{T}(\mathrm{x})$, which corrects for searching across a whole brain volume, is used to localize the region of statistically significant structural displacement (Worsley, 1994).

\section{Important Measures in Brain Development}

We have presented two different statistics, (3) and (5), based on local translation and local volume changes to measures morphological changes over time. One might ask if these two statistics are sufficient to capture temporally varying morphological changes in brain and how one statistic is related to the other. Do they measure common morphological properties or different aspects of morphological changes? In this section, we will give some answers to these questions.
F or relatively small displacement, neglecting higher order terms in the Taylor expansion, the displacement $\mathrm{U}$ at $\mathrm{x}+\mathrm{dx}$ can be written as

$$
U(x+d x, t) \approx U(x, t)+\frac{\partial U}{\partial x}(x, t) d x
$$

As we have pointed out, in tensor-based morphometry some of all elements of the $3 \times 3$ displacement gradient matrix $\partial \mathrm{U} / \partial \mathrm{x}$ are used to measure morphological changes (Thirion and Calmon, 1997; Ashburner et al., 2000; Thompson et al., 2000). The displacement tensor can be further decomposed into two parts depending on whether it is symmetric or antisymmetric:

$$
\frac{\partial U_{j}}{\partial x_{i}}=\frac{1}{2}\left(\frac{\partial U_{j}}{\partial x_{i}}-\frac{\partial U_{i}}{\partial x_{j}}\right)+\frac{1}{2}\left(\frac{\partial U_{j}}{\partial x_{i}}+\frac{\partial U_{i}}{\partial x_{j}}\right) .
$$

The antisymmetric first part corresponds to a rotation or vorticity of the deformation and the symmetric second part corresponds to a strain. Then the displacement at $\mathrm{x}+\mathrm{dx}$ can be decomposed into three parts,

$$
U(x+d x, t) \approx U(x, t)-w(x, t) x d x+\epsilon(x, t) d x
$$






FIG. 4. (L eft) 3D statistical parametric maps of local volume increase (red), volume decrease (blue), and structural displacement (yellow) thresholded at the probability $0.025,0.025,0.05$ (corrected). (Right) Statistical parametric maps are superimposed on the axial, sagittal, and coronal sections of the atlas brain MRI. The cross-sections are taken at the interior of the largest red cluster inside the purple box (somatosensory and motor cortex). The white lines indicate where the cross-sections are taken.

where $\mathrm{w}=\frac{1}{2}(\nabla \times \mathrm{U})$ is the vorticity vector and $\epsilon=$ $\left(\epsilon_{i j}\right)=\frac{1}{2}\left[\partial U / \partial x+(\partial U / \partial x)^{t}\right]$ is the strain matrix. By taking the temporal derivative, we have the displacement velocity decomposed into three parts:

$$
\begin{aligned}
\frac{\partial \mathrm{U}}{\partial \mathrm{t}}(\mathrm{x}+\mathrm{d}, \mathrm{t}) \approx \frac{\partial \mathrm{U}}{\partial \mathrm{t}}(\mathrm{x}, \mathrm{t})-\frac{\partial \mathrm{w}}{\partial \mathrm{t}}(\mathrm{x}, \mathrm{t}) & \times \mathrm{dx} \\
& +\frac{\partial \epsilon}{\partial \mathrm{t}}(\mathrm{x}, \mathrm{t}) \mathrm{dx} .
\end{aligned}
$$

Equation (6) captures most of the spatiotemporal variabilities of the displacement velocity into three compo- nents: the rate of changes in a translation, a rotation, and a strain for relatively small displacements.

The strain-rate tensor $\partial \epsilon_{\mathrm{ij}} / \partial \mathrm{t}$ can be further separated into two parts: the diagonal elements $\partial \epsilon_{i j} / \partial t$ describing the length change of the volume element in each $x_{1}, x_{2}$, and $x_{3}$ coordinate, and the off-diagonal elements $\partial \epsilon_{i j} / \partial t(i \neq j)$ describing the shearing rate of the volume element. The volume element is a mathematical abstraction defined as an infinitesimally small cube, but because the smallest unit in brain imaging is a voxel, we may take the voxel as the volume element. Shearing is the deformation that preserves the volume of a voxel but distorts its shape. Note that the sum of 
the diagonal elements of the strain rate is the firstorder approximation to the rate of the Jacobian change; i.e.,

$$
\frac{\partial \mathrm{J}}{\partial \mathrm{t}} \approx \frac{\partial \mathrm{K}}{\partial \mathrm{t}}=\frac{\partial \epsilon_{11}}{\partial \mathrm{t}}+\frac{\partial \epsilon_{22}}{\partial \mathrm{t}}+\frac{\partial \epsilon_{33}}{\partial \mathrm{t}} .
$$

It seems that we may have to consider translational, rotational, and strain changes for a complete morphological description. However, the most meaningful measurement of brain tissue growth or loss is the rate of the J acobian change because it directly measures the volumetric changes in the brain. The local translation, the local rotation, and the local shearing change can all be considered as readjustments and reorientations of the local brain structure due to the volumetric changes in the neighboring regions (Fig. 2). In between-subject morphological studies of different clinical populations, such measurements might be useful criteria of shape differences. However, in temporally varying within-subject brain morphological studies, we are more interested in regions of brain tissue growth or loss that cause the volumetric changes. Hence, the rate of the J acobian change is the most meaningful morphological measure of brain tissue growth or loss in deformation-based morphometry.

Finally, the dilatation statistic that consists of spatial derivatives of the displacement field is statistically independent from the local translation statistic. To see this, note that any partial derivative of a stationary Gaussian random field is statistically independent from the field itself (Adler, 1981). Since the dilatation consists of spatial derivatives of the displacement, it must be statistically independent of the displacement. So Hotelling's $T^{2}$ field of the displacement and the $T$ field of the dilatation measure morphologically different properties at the same voxel.

\section{Detecting Global Volume Change}

Standard MRI-based volumetry, where we are interested in detecting volume changes of the regions of interest (ROI), can be considered a special case of deformation-based volumetry. Let $\Omega_{t}^{\text {ROI }}$ be the 3D region of interest with smooth $2 \mathrm{D}$ boundary $\partial \Omega_{\mathrm{t}}^{\mathrm{ROI}}$ at time t. The region $\Omega_{0}^{\mathrm{ROI}}$ deforms to $\Omega_{\mathrm{t}}^{\mathrm{ROI}}$ under the deformation $\mathrm{x} \rightarrow \mathrm{x}+\mathrm{U}(\mathrm{x}, \mathrm{t})$. N ote that the volume of $\Omega_{\mathrm{t}}^{\mathrm{ROI}}$ is given by

$$
\left\|\Omega_{\mathrm{t}}^{\mathrm{ROI}}\right\|=\int_{\Omega_{\mathrm{t}}^{\mathrm{ROI}}} \mathrm{dx}=\int_{\Omega_{0}^{\mathrm{ROI}}} \mathrm{J}(\mathrm{x}, \mathrm{t}) \mathrm{dx} .
$$

Then the ROI volume-dilatation rate $\Lambda_{\mathrm{RO}}$ is given by

$$
\begin{aligned}
\Lambda_{\mathrm{ROI}} & =\frac{1}{\left\|\Omega_{0}^{\mathrm{ROI}}\right\|} \frac{\partial}{\partial \mathrm{t}}\left\|\Omega_{\mathrm{t}}^{\mathrm{ROI}}\right\| \\
& =\frac{1}{\left\|\Omega_{0}^{\mathrm{ROI}}\right\|} \int_{\Omega_{0}^{\mathrm{ROI}}} \frac{\partial \mathrm{J}}{\partial \mathrm{t}} \mathrm{dx} \\
& \approx \frac{1}{\left\|\Omega_{0}^{\mathrm{ROI}}\right\|} \int_{\Omega_{0}^{\mathrm{ROI}}} \Lambda_{\text {volume }} \mathrm{dx} .
\end{aligned}
$$

Therefore, the global ROI volume dilatation rate $\Lambda_{\mathrm{RO}}$ is equivalent to the average of the local volume dilatation rate $\Lambda_{\text {volume }}$ taken over all $\Omega_{0}^{\mathrm{ROI}}$. Since $\Lambda_{\text {volume }}$ is distributed as a Gaussian random field, $\Lambda_{\text {ROI }}$ becomes a Gaussian random variable. So testing the hypothesis whether there is any volume change between $\Omega_{0}^{\mathrm{ROI}}$ and $\Omega_{\mathrm{t}}^{\mathrm{ROI}}$ can be performed through a simple t test.

It is also possible to test the global volume change via surface-based deformation analysis. Gauss's Divergence Theorem states that

$$
\int_{\Omega_{0}^{\mathrm{ROI}}} \nabla \cdot \mathrm{Udx}=\int_{\partial \Omega_{0}^{\mathrm{ROI}}} \mathrm{U} \cdot \mathbf{n} \mathrm{dA},
$$

where $\mathbf{n}$ is a unit normal vector on the surface $\partial \Omega_{0}^{\text {ROI }}$ and $\mathrm{dA}$ is the surface area element (Marsden and Hughes, 1983). It follows that

$$
\Lambda_{\mathrm{ROI}} \approx \frac{1}{\left\|\Omega_{0}^{\mathrm{ROI}}\right\|} \int_{\partial \Omega_{0}^{\mathrm{ROI}}} \mathrm{V} \cdot \mathbf{n d A},
$$

where $\mathrm{V}=\partial \mathrm{U} / \partial \mathrm{t}$ is the surface displacement velocity defined on the boundary $\partial \Omega_{0}^{\mathrm{ROI}}$. Since $V$ is distributed as a Gaussian random field, $\Lambda_{\text {ROI }}$ is distributed as a Gaussian random variable and statistical inference will be again based on a simple test.

\section{RESULTS}

Twenty-eight normal subjects were selected based on the same physical, neurological, and psychological criteria described in Giedd et al. (1996a). Two $\mathrm{T}_{1^{-}}$ weighted MR scans were acquired for each subject at different times on the same GE Sigma 1.5-T superconducting magnet system. The first scan was obtained at the age of $11.5 \pm 3.1$ years (min 7.0 years, max 17.8 years) and the second scan was obtained at the age of $16.1 \pm 3.2$ years ( $m i n 10.6$ years, max 21.8 years). The time difference between the first and the second scan was $4.6 \pm 0.9$ years ( $\mathrm{min}$ time difference 2.2 years, max 
time difference 6.4 years). Using the automatic imageprocessing pipeline (Zijdenbos et al., 1998), a total of 56 MR images were transformed into standardized stereotactic space via a global affine transformation (Talairach and Tournoux, 1988) followed by a nonlinear deformation to match the atlas brain $\Omega_{\text {atlas. }}$. The global affine transformation removes most of the intra- and intersubject global differences in brain sizes; adult brains are approximately 5\% larger than those of 5-year-old children (Dekaban, 1977; Dekaban and Shadowsky, 1978). Because we are only interested in finding local morphological changes, these global morphological variabilities should be removed via global affine transform in order to improve the power of detection. These registration procedures are based on an automatic multiresolution intensity matching algorithm (Collins et al., 1995; Collins and Evans, 1999). Unlike other registration algorithms that assume a certain fluid dynamics or an elastic deformation model, the intensity-based registration does not assume any explicit physical model in which the deformation from the subject brain to the atlas brain should follow (Gee and Bajcsy, 1999; Thompson et al., 2000). So the deformation fields obtained from these registration processes can be considered free of any explicit physical model assumption although there might be some intensity-based model assumption, which somehow relates to a physical model.

If $U_{S 1}^{j}$ and $U_{S 2}^{j}$ are the displacement obtained from the nonlinear registration of the first and the second scan of subject $\mathrm{j}$ to the atlas brain $\Omega_{\text {atlas }}$ at time $\mathrm{t}_{\mathrm{s} 1}^{\mathrm{j}}$ and $\mathrm{t}_{\mathrm{s} 2}^{\mathrm{j}}$, the actual displacement $\mathrm{U}^{\mathrm{j}}$ between the first scan and the second scan is $U^{j}=U_{S 1}^{j}-U_{S 2}^{j}$ and the time difference is $t_{j}=t_{s 2}^{j}-t_{s 1}^{j}$ (Fig. 3). It is true that if the first scan were directly registered to the second scan without going through the atlas brain, the registration error would be smaller. However, the displacement fields obtained by the direct registration method still must be registered onto the atlas brain in order to form statistical parametric maps. The reason for such statistical treatment to analyze the structural data is obvious considering that the displacement field obtained from image registration algorithms for brain devel opment contains a fairly large component of error. The length of the displacement velocity we have observed for the spatially normalized MR scans of 28 normal subjects is usually less than $1 \mathrm{~mm} /$ year, i.e., $\mu_{0}=\mathbb{E}(\partial \mathrm{U} / \partial \mathrm{t}) \leq 1 \mathrm{~mm} /$ year in average. Optimistically assuming that the image registration algorithm is accurate to within one voxel distance (usually 1 or $2 \mathrm{~mm}$ ), the registration error seems to be relatively large in brain development. So one may be skeptical about whether the deformation-based morphometry can possibly detect such small changes. Nevertheless it is still possible to pick out the signal when there are enough data; Figure 1 illustrates how image smoothing and the statistical treatments improve the power of detec- tion. Statistical treatments compensate for some of such registration errors. Finally the displacement velocity field is smoothed with a $10 \mathrm{~mm}$ full width at half-maximum (FWHM) Gaussian kernel to increase the signal to noise ratio. Gaussian kernel smoothing with $\mathrm{FWHM}=4(\ln 2)^{1 / 2} \sqrt{t}$ of the signal $f(x), x \in \mathbb{R}^{3}$ is defined as the convolution of the signal $f$ with the Gaussian kernel:

$$
F(x, t)=\frac{1}{(4 \pi t)^{3 / 2}} \int_{\mathbb{R}^{3}} e^{-(x-y)^{2} / 4 t} f(y) d y
$$

Without the smoothing, it may have been more difficult to detect morphological patterns illustrated in Fig. 1. However, the Gaussian kernel smoothing sometimes tends to blur the fine details of deformation pattern.

The regions of statistically significant displacement have been detected (Fig. 4, yellow) by Hotelling's $\mathrm{T}^{2}$ field with the corrected threshold (Cao and Worsley, 1999):

$$
\mathrm{P}\left(\max _{\mathrm{x} \in \Omega_{\text {atlas }}} \mathrm{H}(\mathrm{x})>60.0\right) \approx 0.05 \text {. }
$$

Most of the structural movements were observed in the frontal lobe without any accompanying significant change in local volume. This may indicate that there are continued readjustments of the exact position of brain structures in the frontal lobe without any brain tissue growth or loss in adolescence. Also note that the statistically significant displacement occurs evenly and shows some degree of symmetry between the left and the right hemispheres. Because the local translation statistic measures the relative displacement of brain structure, it does not truly reflect the brain tissue growth process. However, it does indicate the principal direction of the brain growth as shown in the purple box in Fig. 4 and enlarged in Fig. 5. Hence, the local translation statistic should be used in conjunction with the local volume change statistic to fully understand the complex dynamics of temporally changing morphological pattern.

Previous developmental MRI studies have provided evidence for age-related increase in total white matter volume and decrease in total gray matter volume (J ernigan et al., 1991; Pfefferbaum et al., 1994; Rajapakse et al., 1996; Riess et al., 1996; Courchesne et al., 2000), but the analytic procedures used in these studies did not allow the investigators to detect local volume change. The local volume change statistic $T(x)$ is computed using the formula (5) with $t_{j}=t_{s 1}^{j}-t_{s 2}^{j}$. The t statistic map is thresholded at

$$
\begin{array}{r}
\mathrm{P}\left(\max _{\mathrm{x} \in \Omega_{\text {atlas }}} \mathrm{T}(\mathrm{x})>6.5\right) \approx 0.025, \\
\mathrm{P}\left(\min _{\mathrm{x} \in \Omega_{\text {atlas }}} \mathrm{T}(\mathrm{x})<-6.5\right) \approx 0.025 .
\end{array}
$$




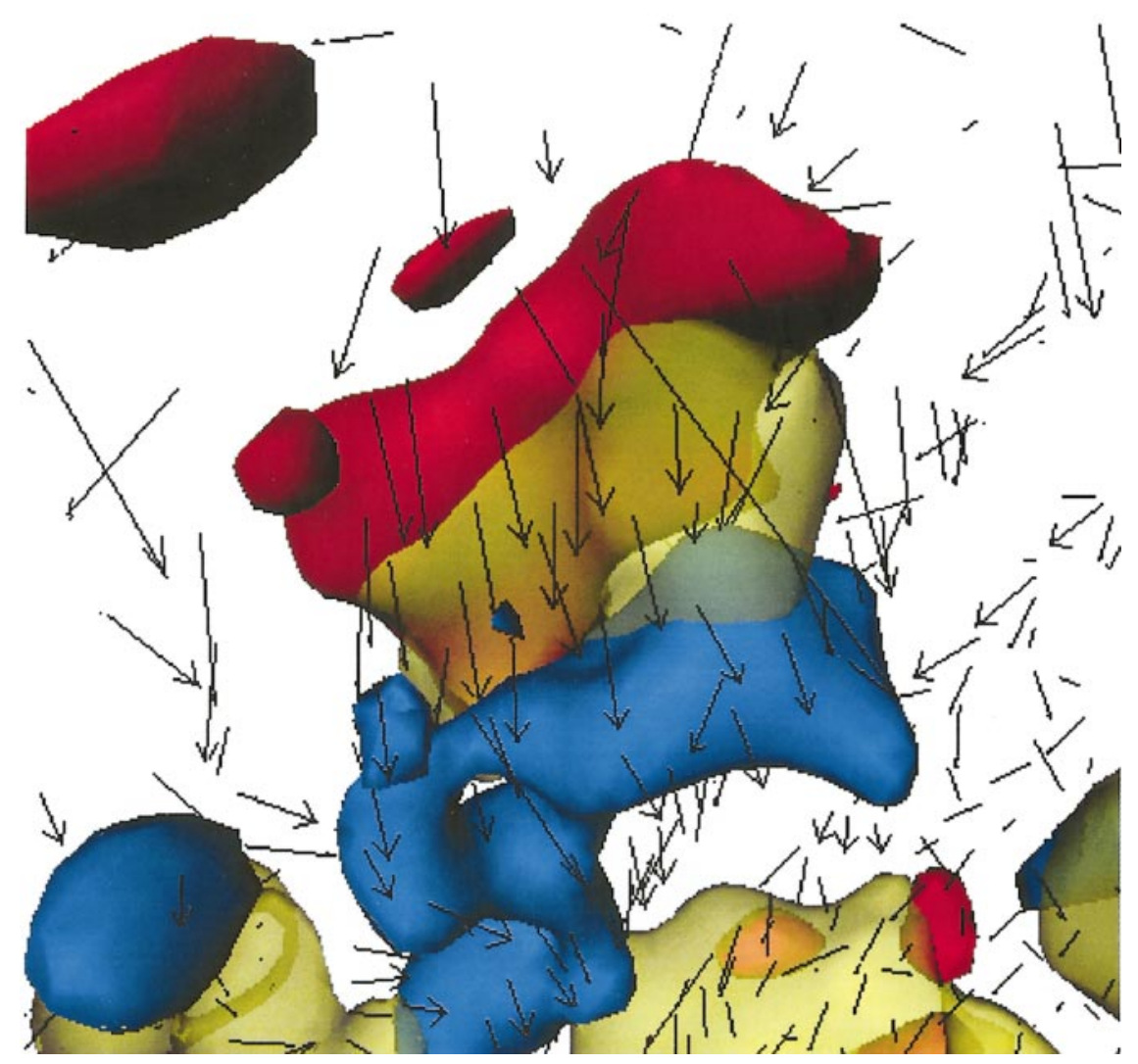

FIG. 5. A close-up of part of the outer left hemisphere inside the purple box in Fig. 4. Black arrows represent the sample mean displacement velocity subsampled every $10 \mathrm{~mm}$ and scaled by $50 \mathrm{~mm} /$ year. The direction of the mean displacement vel ocity suggests how the local volume expansion (red) causes the translational movement of the structure (yellow) toward the region of atrophy (blue). The heads of arrows are manually enhanced to clearly indicate the direction of the displacement.

At this threshold, most of the local volume increase observed around the corpus callosum in Fig. 1 disappears except for very few localized statistically significantly "peaks" in the isthmus and splenium. There was no volume change detected in the rostrum and genu. Figure 4 also shows the localized growth in the corpus callosum on the coronal section (the single red dot). Therefore, we observe a highly focused region of brain tissue growth in the isthmus and splenium of the corpus call osum. Pujol et al. (1993), Giedd et al. (1996b, 1999), and Thompson et al. (2000) reported similar results of growth pattern at the corpus callosum.

The growth at the corpus callosum seems relatively small when compared to the global peaks observed predominantly in somatosensory and motor cortex (the largest red cluster in Fig. 4). Localized brain tissue loss was also detected at the same time as tissue growth. This tissue loss was highly localized in the subcortical region of the left hemisphere (Fig. 4, blue). Similar results were also reported in Thompson et al. (2000), where the extent of the peak growth was wider and less localized than our study has found. It seems our statistical treatments based on the large sample size $(\mathrm{n}=$ 28) tend to remove a lot of intrasubject variabilities and pick out the common morphological pattern among subjects compared to the smaller sample size $(n=6)$ studied in Thompson et al. (2000). Slightly different growth patterns observed between our study and Thompson et al. (2000) may be due to many factors. Our approach is based on the systematic statistical treatments of large sample size $(n=28)$ with a less accurate intensity-based automatic registration algorithm. While the approach taken in Thompson et al. (2000) is based on a sample size of six without any statistical approach, a more accurate elastic model based registration algorithm with manually matched sulcal landmarks was used. However, the most important difference between the two studies is the age distribution of the subjects. In Thompson et al. (2000), the age distribution of the six subjects is in most part younger than our mean age of 11.5 years for the first scan and 16.1 years for the second scan. So although there are similar growth patterns common to both studies such as predominant growth at parietal cortex, localized peak growth at the corpus callosum, etc., the two studies are detecting morphological changes in different but nonexclusive age groups.

We have observed very interesting relations between local displacement change and local volume change statistics as illustrated in Figs. 4 and 5 . Figure 5 is the 
close-up of the parietal region of the left hemisphere (the purple box in Fig. 4), showing a large local displacement from the region of local volume increase (gray matter) to a region of local volume decrease (white matter), indicating how the structure boundary (inner cortical surface) has moved from the increasing volume to the decreasing volume. This phenomenon is also schematically illustrated in Fig. 1b, where the square grid is undergoing a horizontal translation from the region of volume increase of the left to the region of volume decrease on the right, and Fig. $2 d$, where the volume expansion in the middle causes the neighboring structures to radially translate outward. It seems that by studying these two statistical parametric maps simultaneously, the complex dynamic patterns in temporally varying brain morphology can be captured.

\section{CONCLUSIONS}

The deformation-based volumetry presented here can localize the regions where local volume growth or loss occurs over temporally varying brain morphology by measuring the rate of local volume changes. By using the displacement vel ocity instead of the displacement itself in detecting the anatomical changes, temporal variabilities in MR images for different age groups and different time intervals can be accounted for. As an illustration, we have applied the method to MR scans of 28 normal children and adolescents and detected regions of tissue growth in the corpus callosum and somatosensory and motor cortex.

Our unified statistical framework based on the deformation-based volumetry can be also used as a tool for future investigations of neurodevelopmental disorders where volumetric analysis would be relevant. It can also be applied to a general morphological studies, such as testing for structural shape differences between two different groups of subjects.

\section{ACKNOWLEDGMENTS}

Steve Robbins at Montreal Neurological Institute provided technical assistance with the atlas brain. The valuable comments of J ohn Ashburner at the Wellcome Department of Cognitive Neurology; Luke Oh at the Department of Neuroscience, University of Connecticut Health Center; and J im Ramsay at the Department of Psychology, McGill University, are also acknowledged. The Matlab program used in computing the corrected thresholds of the T random field and Hotelling's $T^{2}$ random field can be found at http://www.math. mcgill.ca/keith/BICstat.

\section{REFERENCES}

Adler, R. J . 1981. The Geometry of Random Fields, p. 31. Wiley, New York.

Ashburner, J ., Neelin, P., Collins, D. L., Evans, A. C., and Friston, K.J . 1997. Incorporating prior knowledge into image registration. Neurol mage 6: 344-352.
Ashburner, J ., and Friston, K. J . 2000. Voxel-based morphometryThe methods. Neurol mage 11: 805- 821.

Ashburner, J ., Good, C., and Friston, K. J . 2000. Tensor-based morphometry. Neurol mage 11S: 465.

Cao, J ., and Worsley, K. J . 1999. The detection of local shape changes via the geometry of Hotelling's T ${ }^{2}$ fields. Ann. Stat. 27: 925-942.

Christensen, G. E., Rabbitt, R. D., and Miller, M. I. 1993. A deformable neuroanatomy text-book based on viscous fluid mechanics. In Proceedings of the 27th Annual Conference on Information Science and Systems, pp. 211-216.

Christensen, G. E., J oshi, S. C., and Miller, M. I. 1997. Volumetric transformation of brain anatomy. IEEE Trans. Med. Imag. 16: 864-877.

Collins, D. L., Holmes, C. J ., Peters, T. M., and Evans, A. C. 1995. Automatic 3D model-based neuroanatomical segmentation. Hum. Brain Map. 3: 190-208.

Collins, D. L., Paus, T., Zijdenbos, A., Worsley, K. J ., Blumenthal, J ., Giedd, J . N., Rapoport, J . L., and Evans, A. C. 1998. Age related changes in the shape of temporal and frontal lobes: An MRI study of children and adolescents. Soc. Neurosci. Abstr. 24: 304.

Collins, D. L., and Evans, A. C. 1999. ANIMAL: Automatic nonlinear image matching and anatomical labeling. In Brain Warping, pp. 133-142. Academic Press, San Diego.

Courchesne, E., Chisum, H. J ., Townsend, J ., Cowles, A., Covington, J ., Egaas, B., Harwood, M., Hinds, S., and Press, G. A. 2000. Normal brain development and aging: Quantitative analysis at in vivo MR imaging in healthy volunteers. Radiology 216: 672- 682.

Davatzikos, C., Vaillant, M., Resnick, S. M., Prince, J . L., Letovsky, S., and Bryan, N. 1996. A computerized approach for morphological analysis of the corpus callosum. J . Comput. Assist. Tomogr. 20: 88-97.

Davatzikos, C. 1999. Brain morphometrics using geometry-based shape transformations. In Proceedings of Workshop on Biomedical I mage Registration, Slovenia.

Dekaban, A. S. 1977. Tables of cranial and orbital measurements, cranial volume and derived indexes in males and females from 7 days to 20 years of age. Ann. Neurol. 2: 485- 491.

Dekaban, A. S., and Shadowsky, D. 1978. Changes in brain weights during the span of human life: Relation of brain weights to body heights and body weights. Ann. Neurol. 4: 345-356.

Gaser, C., Volz, H.-P., Kiebel, S., Riehemann, S., and Sauer, H. 1999. Detecting structural changes in whole brain based on nonlinear deformations-Application to schizophrenia research. Neurol mage 10: 107-113.

Gee, J. A., and Bajcsy, R. K. 1999. Elastic matching: Continuum mechanical and probabilistic analysis. In Brain Warping, pp. 183198. Academic Press, San Diego.

Giedd, J . N., Snell, J. W., Lange, N., Rajapakse, J . C., Kaysen, D., Vaituzis, A. C., Vauss, Y. C., Hamburger, S. D., Kozuch, P. L., and Rapoport, J . L. 1996a. Quantitative magnetic resonance imaging of human brain development: Ages 4-18. Cereb. Cortex 6: 551-160.

Giedd, J. N., Rumsey, J. M., Castellanos, F. X., Rajapakse, J . C., Kaysen, D., Vaituzis, A. C., Vauss, Y. C., Hamburger, S. D., and Rapoport, J. L. 1996b. A quantitative MRI study of the corpus callosum in children and adolescents. Dev. Brain Res. 91: 274-280.

Giedd, J. N., Blumenthal, J ., J effries, N. O., Rajapakse, J . C., Vaituzis, A. C., Liu, H., Berry, Y. C., Tobin, M., Nelson, J., and Castellanos, F. X. 1999. Development of the human corpus callosum during childhood and adolescence: A Iongitudinal MRI study. Prog. Neuro-Psychopharmacol. Biol. Psychiatry 23: 571-588.

J ernigan, T. L., Trauner, D. A., Hesselink, J. R., and Tallal, P. A. 1991. Maturation of human cerebrum observed in vivo during adolescence. Brain 114: 2037-2049. 
J oshi, S. C. 1998. LargeDeformation Diffeomorphisms and Gaussian Random Fields for Statistical Characterization of Brain Sub-Manifolds, Ph.D. thesis. Washington University, St. Louis.

Landau, L. D., and Lifshitz, E. M. 1989. Fluid Mechanics, 2nd ed., Course of Theoretical Physics, Vol. 6, pp. 44-51. Pergamon, Elmsford, NY.

Marsden, J., and Hughes, T. 1983. Mathematical Foundations of Elasticity. Dover, New York.

Pfefferbaum, A., Mathalon, D. H., Sullivan, E. V., Rawles, J. M., Zipursky, R. B., and Lim, K. O. 1994. A quantitative magnetic resonance imaging study of changes in brain morphology from infancey to late adulthood. Arch. Neurol. 51: 874- 887.

Pujol, J ., Vendrell, P., J unque, C., Martivilalta, J . M., and Capdevila, A. 1993. When does human brain development end? Evidence of corpus callosum growth up to adulthood. Ann. Neurol. 34: 71-75.

Rajapakse, J . C., Giedd, J . N., DeCarli, C., Snell, J . W., McLaughlin, A., Vauss, Y. C., Krain, A. L., Hamburger, S., and Rapoport, J . L. 1996. A technique for single-channel MR brain tissue segmentation: Application to a pediatric sample. Magn. Reson. Imag. 14: 1053-1065.

Riess, A. L., Abrams, M. T., Singer, H. S., Ross, J . L., and Denckla, M. B. 1996. Brain development, gender and IQ in children: A volumetric imaging study. Brain 119: 1763-1774.

Talairach, J ., and Tournoux, P. 1988. Co-planar Stereotactic Atlas of the Human Brain-3-Dimensional Proportional System: An Approach to Cerebral Imaging. Thieme, Stuttgart.

Thirion, J .-P., and Calmon, G. 1999. Deformation analysis to detect quantify active lesions in $3 D$ medical image sequences. IEEE Trans. Med. Imag. 18: 429-441.

Thompson, P. M., and Toga, A. W. 1999. Anatomically driven strategies for high-dimensional brain image warping and pathology detection. In Brain Warping, pp. 311-336. Academic Press, San Diego.

Thompson, P. M., Giedd, J. N., Woods, R. P., MacDonald, D., Evans, A. C., and Toga, A. W. 2000. Growth patterns in the developing human brain detected using continuum-mechanical tensor mapping. Nature 404: 190-193.

Thompson, P. M., MacDonald, D., Mega, M. S., Holmes, C. J ., Evans, A. C., and Toga, A. W. 1997. Detection and mapping of abnormal brain structure with a probabilistic atlas of cortical surfaces. J . Comput. Assist. Tomogr. 21: 567-581.

Toga, A. W., Thompson, P. M., and Payne, B. A. 1996. Modeling morphometric changes of the brain during development. In De velopmental Neuroimaging, pp. 15-27. Academic Press, San Diego.

Warfield, S., Robatino, A., Dengler, J ., J olesz, F., and Kikinis, R. 1999. Nonlinear registration and template-driven segmentation. In Brain Warping, pp. 67-84. Academic Press, San Diego.

Worsley, K. J . 1994. Local maxima and the expected Euler characteristic of excursion sets of $\chi^{2}, \mathrm{~F}$ and $\mathrm{t}$ fields. Adv. Appl. Probabil. 26: $13-42$.

Worsley, K. J . 1996. An Unbiased Estimator for the Roughness of a Multivariate Gaussian Random Field, technical report. Department of Mathematics and Statistics, McGill University. http: IIwww.math.mcgill.cakkeith.

Worsley, K. J ., Marrett, S., Neelin, P., Vandal, A. C., Friston, K. J ., and Evans, A. C. 1996. A unified statistical approach for determining significant signals in images of cerebral activation. Hum. Brain Map. 4: 58-73.

Zijdenbos, A. P., J imenez, A., and Evans, A. C. 1998. Pipelines: Large scale automatic analysis of 3D brain data sets. Neurol mage 7S: 783. 